\title{
The challenges and opportunities for
} \section{implementation of eDNA biomonitoring in riverine}

\section{systems}

\author{
Florian Altermatt $\ddagger$ \\ ‡ Eawag, Duebendorf, Switzerland
}

Corresponding author: Florian Altermatt (florian.altermatt@eawag.ch)

Received: 04 Mar 2021| Published: 04 Mar 2021

Citation: Altermatt F (2021) The challenges and opportunities for implementation of eDNA biomonitoring in riverine systems. ARPHA Conference Abstracts 4: e65521. https://doi.org/10.3897/aca.4.e65521

\begin{abstract}
Current local to global threats to biodiversity and anthropogenic changes of the environment call for rapid and effective conservation and management of ecosystems and the services they provide. In this context, the use of environmental DNA to assess biodiversity and conduct biomonitoring has been established as a novel, potentially revolutionizing approach over the last decade, especially in aquatic ecosystems. Rapid initial success, broad applicability and advances in sequencing technologies have raised high expectations about its potential. However, as with any revolution, true success requires formal implementation and establishing and integration of routines. In this talk, I will exemplify challenges and opportunities for implementation of eDNA biomonitoring in riverine systems, addressing both scientists and stakeholders. I will discuss potential pitfalls and misunderstandings caused by different targets, inference, and possible conclusions when comparing traditional sampling approaches with eDNA. I postulate that the focus should be on the strengths of new approaches, and not their matching to existing techniques. Finally, I will exemplify a strategy and the necessary steps of how a new order in biomonitoring can be established.
\end{abstract}

\section{Presenting author}

Florian Altermatt 


\section{Presented at}

1st DNAQUA International Conference (March 9-11, 2021) 